\title{
Incorporating Backbone Flexibility in MedusaDock Improves Ligand-Binding Pose Prediction in the CSAR2011 Docking Benchmark
}

${ }_{4}$ Feng Ding ${ }^{\dagger, \ddagger}$ and Nikolay V. Dokholyan*, ${ }^{\ddagger}$

$5{ }^{\dagger}$ Department of Physics and Astronomy, Clemson University, Clemson, South Carolina 29634, United States

$6{ }^{\ddagger}$ Department of Biochemistry and Biophysics, University of North Carolina at Chapel Hill, School of Medicine, Chapel Hill,

7 North Carolina 27599, United States
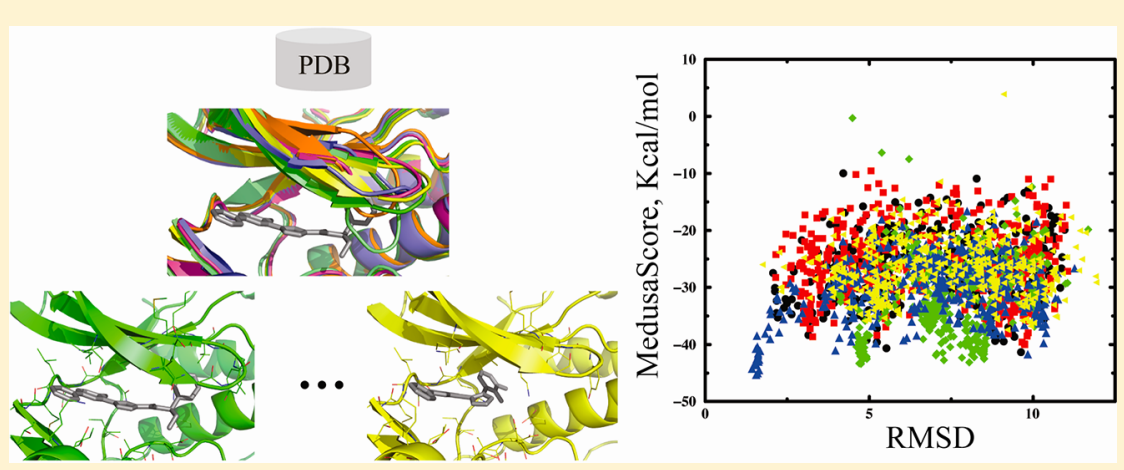

ABSTRACT: Solution of the structures of ligand-receptor complexes via computational docking is an integral step in many structural modeling efforts as well as in rational drug discovery. A major challenge in ligand-receptor docking is the modeling of both receptor and ligand flexibilities in order to capture receptor conformational changes induced by ligand binding. In the molecular docking suite MedusaDock, both ligand and receptor side chain flexibilities are modeled simultaneously with sets of discrete rotamers, where the ligand rotamer library is generated "on the fly" in a stochastic manner. Here, we introduce backbone flexibility into MedusaDock by implementing ensemble docking in a sequential manner for a set of distinct receptor backbone conformations. We generate corresponding backbone ensembles to capture backbone changes upon binding to different ligands, as observed experimentally. We develop a simple clustering and ranking approach to select the top poses as blind predictions. We applied our method in the CSAR2011 benchmark exercise. In 28 out of 35 cases (80\%) where the ligand-receptor complex structures were released, we were able to predict near-native poses $(<2.5 \AA$ RMSD), the highest success rate reported for CSAR2011. This result highlights the importance of modeling receptor backbone flexibility to the accurate docking of ligands to flexible targets. We expect a broach application of our fully flexible docking approach in biological studies as well as in rational drug design.

\section{INTRODUCTION}

22 One major challenge in computational prediction of receptor23 ligand interactions is the large number of degrees of freedom, 24 including receptor backbone and side chain flexibilities, ligand 25 conformational flexibility, and ligand rigid-body motion. Of par26 ticular interest is receptor flexibility, which is essential for cap27 turing the receptor conformational changes upon ligand bind28 ing, i.e., the induced-fit effect. ${ }^{1-5}$ Receptor induced-fit can be 29 limited to the rearrangement of side chains in the binding pocket, 30 or extended to major rearrangement of the backbone, as observed 31 in many kinases. ${ }^{6}$ Because of the high dimensionality in describing 32 receptor conformational changes, modeling receptor flexibility is 33 highly challenging and has been one of the foci of recent ligand34 receptor docking studies. ${ }^{3-5,7-11}$

35 Several approaches have been proposed to capture receptor con36 formational changes. For example, the generation of an ensemble of 37 multiple predetermined conformations has been proposed to model 38 the receptor flexibility. The receptor conformation ensemble can be obtained experimentally by X-ray crystallography under differ- 39 ent conditions or by NMR spectroscopy, ${ }^{12-15}$ computationally 40 by molecular dynamics simulations, ${ }^{7,16-19}$ comparative model- 41 ing, ${ }^{20}$ or normal-mode analysis. ${ }^{11,21}$ In these approaches, the 42 derivation of structural ensembles representing binding-induced 43 receptor conformational change is decoupled from the modeling 44 of ligand binding. Each generated structure of the receptor is 45 kept rigid during ligand docking, and receptor conformational 46 flexibility is realized by selecting the optimal poses from ensemble 47 docking in either sequential (independent) ${ }^{22}$ or coupled ${ }^{23}$ man- 48 ners. Therefore, the major challenge to overcome in using these 49 approaches is that the predetermined receptor conforma- 50 tional ensemble must encompass significant sampling such 51

Special Issue: 2012 CSAR Benchmark Exercise

Received: October 6, 2012 
Table 1. Summary Table of the Predicted Ligand-kinase Poses. ${ }^{a}$

\begin{tabular}{|c|c|c|c|c|c|c|c|}
\hline \multirow[t]{2}{*}{ Kinase } & \multirow{2}{*}{$\begin{array}{l}\text { Ligand } \\
\text { index }\end{array}$} & \multicolumn{3}{|c|}{ Ranked by free energy } & \multicolumn{3}{|c|}{ Ranked by average energy } \\
\hline & & Pose \#1 & Pose \#2 & Pose \#3 & Pose \#1 & Pose \#2 & Pose \#3 \\
\hline \multirow[t]{15}{*}{ chk1 } & 1 & 0.63 & 8.46 & 3.88 & 0.63 & 8.46 & 3.90 \\
\hline & 3 & 0.77 & 10.37 & 9.81 & 0.77 & 10.37 & 9.81 \\
\hline & 4 & 2.03 & 10.33 & 8.86 & 2.03 & 10.33 & 8.86 \\
\hline & 6 & 0.66 & 10.68 & 10.26 & 0.66 & 10.68 & 10.26 \\
\hline & 18 & 1.84 & 6.61 & 3.48 & 1.84 & 6.61 & 3.21 \\
\hline & 20 & 5.84 & 1.01 & 4.09 & 5.84 & 1.01 & 4.09 \\
\hline & 24 & 3.84 & 2.09 & 4.74 & 3.84 & 4.74 & 2.09 \\
\hline & 27 & 7.98 & 1.98 & 8.07 & 7.98 & 9.47 & 1.98 \\
\hline & 29 & 9.03 & 6.91 & 4.69 & 9.03 & 4.69 & 6.91 \\
\hline & $29 *$ & 5.90 & 2.27 & 8.29 & 5.90 & 10.00 & 6.65 \\
\hline & 31 & 6.36 & 6.60 & 1.27 & 1.27 & 6.36 & 6.60 \\
\hline & 34 & 3.50 & 3.95 & 9.27 & 0.52 & 3.50 & 8.21 \\
\hline & 35 & 1.80 & 8.97 & 7.89 & 7.89 & 4.04 & 1.80 \\
\hline & 36 & 1.67 & 6.26 & 6.23 & 1.67 & 4.78 & 4.60 \\
\hline & 37 & 1.12 & 6.68 & 5.88 & 1.12 & 6.68 & 5.11 \\
\hline \multirow[t]{12}{*}{ erk2 } & 19 & 1.49 & 4.71 & 8.31 & 4.71 & 5.24 & 1.49 \\
\hline & 20 & 9.18 & 4.47 & 2.10 & 3.19 & 1.52 & 4.94 \\
\hline & 22 & 2.26 & 7.59 & 6.30 & 8.45 & 2.26 & 8.19 \\
\hline & 23 & 8.62 & 6.25 & 4.07 & 7.22 & 6.19 & 4.07 \\
\hline & 24 & 3.92 & 5.42 & 4.72 & 5.42 & 4.72 & 7.60 \\
\hline & 25 & 7.19 & 5.74 & 5.27 & 5.88 & 8.30 & 4.08 \\
\hline & 26 & 6.52 & 1.09 & 4.98 & 1.09 & 6.86 & 6.52 \\
\hline & 27 & 5.22 & 5.58 & 6.59 & 5.58 & 4.83 & 6.59 \\
\hline & 32 & 2.24 & 9.35 & 6.53 & 9.35 & 9.89 & 9.98 \\
\hline & 33 & 3.45 & 4.38 & 5.49 & 4.38 & 5.49 & 5.31 \\
\hline & 37 & 10.26 & 2.01 & 9.88 & 6.30 & 9.23 & 6.96 \\
\hline & 39 & 5.07 & 1.23 & 5.89 & 5.89 & 6.59 & 5.07 \\
\hline \multirow[t]{5}{*}{ Ipxc } & 2 & 1.33 & 12.07 & 7.19 & 1.33 & 12.07 & 11.04 \\
\hline & 6 & 0.96 & 2.52 & 4.67 & 1.23 & 2.52 & 5.05 \\
\hline & 7 & 1.13 & 2.59 & 10.97 & 1.13 & 2.59 & 5.42 \\
\hline & $7 C$ & 1.44 & 2.73 & 10.98 & 1.44 & 2.73 & 11.04 \\
\hline & 13 & 1.32 & 2.69 & 5.45 & 1.32 & 2.69 & 7.21 \\
\hline \multirow[t]{4}{*}{ urokinase } & 8 & 1.16 & 1.94 & 2.28 & 1.16 & 1.94 & 2.28 \\
\hline & 12 & 2.57 & 0.52 & 2.22 & 2.57 & 0.52 & 2.22 \\
\hline & 14 & 1.26 & 5.15 & 6.37 & 1.26 & 5.15 & 6.37 \\
\hline & 18 & 4.52 & 0.46 & 2.71 & 0.46 & 4.52 & 2.71 \\
\hline
\end{tabular}

${ }^{a}$ For each ligand-receptor pair, three poses are submitted for the CSAR 2011 exercise. Two ranking methods, according to either binding free energy or the average binding energy (Methods section), were used. The RMSD values smaller than $2.5 \AA$ are highlighted in italic bold font. The cases where none of the top three poses are within $2.5 \AA$ RMSD are in gray shading. * The given smile of ligand \#29 for chk 1 was different from the actual ligand. We performed posterior docking simulations using the same ligand as observed in the crystal structure.

52 that the ensemble contains favorable receptor conformations 53 for ligand binding.

54 Alternative approaches have been proposed to simultane55 ously sample the receptor and ligand flexibilities during 56 docking. ${ }^{8-10,24-27}$ For example, protein side chain rotamer 57 libraries, where continuous protein side chain conformational 58 space is modeled by a set of discrete states, ${ }^{28}$ have been used to 59 model protein flexibility during docking. ${ }^{8,10,24-26}$ Among these 60 rotamer-based approaches, approaches like RosettaLigand ${ }^{9,26}$ 61 and MedusaDock ${ }^{27}$ extensively sample receptor side chain con62 formations near the binding pocket during docking, which has 63 been found to increase the prediction accuracy for near-native 64 poses. Specifically, MedusaDock treats ligand conformational 65 flexibility in the same manner as that of protein side chains, 66 with sets of discrete rotamers. The rotamer library of a ligand is 67 generated in a stochastic manner during docking. Benchmark 68 studies of MedusaDock suggested that sampling protein side 69 chain rotamers together with ligand during docking can effi70 ciently capture the receptor induced-fit, as well as improved 71 virtual screening enrichment for flexible targets. ${ }^{27}$

72 In this study, we incorporate backbone flexibility into 73 MedusaDock in order to blindly predict the ligand-binding poses for the CSAR2011 docking benchmark (www.csardock.org), which 74 includes kinase targets known to be highly flexible. ${ }^{6,8,29,30}$ We adopt 75 a simple multiple backbone conformation docking approach, where 76 an ensemble of backbone conformations is selected to capture the 77 backbone changes as observed in receptor structures solved under 78 different conditions, including binding with different ligands. We 79 then sequentially dock the ligand to the predetermined backbone 80 conformations using the flexible side chain/flexible ligand docking 81 suite, MedusaDock. We cluster the top-ranked poses generated 82 from flexible docking to each backbone conformation in order 83 to group structurally similar predictions. We score and rank the 84 clusters in order to select the optimal ligand-binding poses for 85 CSAR2011 predictions. Using the flexible backbone docking proto- 86 col of MedusaDock, we were able to predict the near-native poses 87 for 28 out of 35 ligands in the CSAR2011 benchmark, the highest 88 success rate of near-native pose predictions ( $<2.5 \AA$ RMSD), which 89 highlights the importance of modeling receptor backbone in accu- 90 rate docking of ligands to a flexible target.

\section{METHODS}

91

MedusaDock. We use MedusaDock ${ }^{27}$ to generate ligand- 93 receptor binding poses. MedusaDock is a flexible docking method, 94 


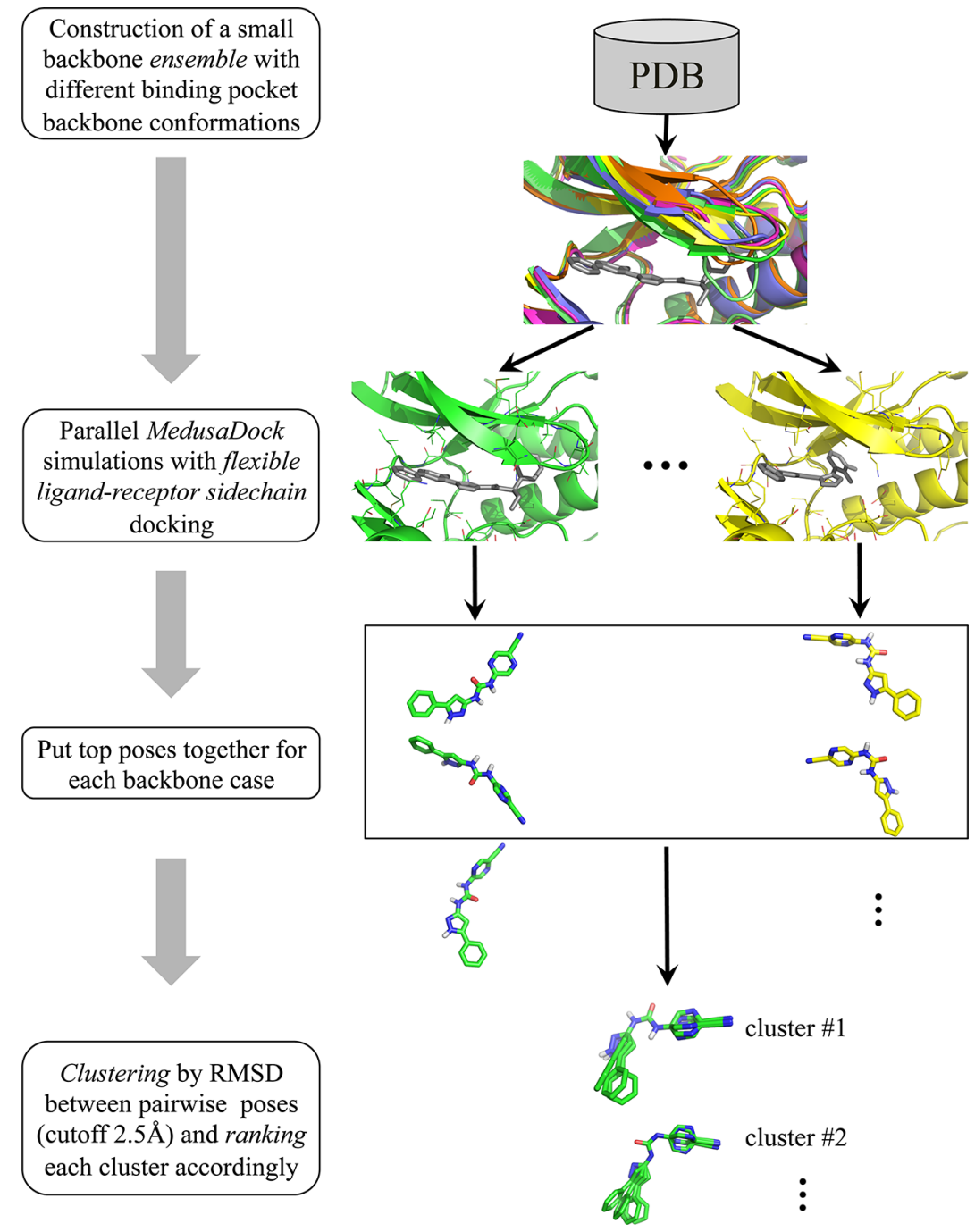

Figure 1. Flowchart of the flexible backbone docking protocol of MedusaDock. The boxes and arrows on the left column summarize the four docking steps (Methods section). The illustrations with protein and/or ligand structures on the right column demonstrate the corresponding steps. Multiple protein backbones in cartoon representation with different colors are selected for independent MedusaDock simulations. The ligands in gray stick are placed in the pocket, where the conformational flexibility of both ligands and receptor side chains (in line representations) are sampled simultaneously. The top poses ranked according MedusaScore are gathered (as shown in the box) for further clustering analysis to group similar poses.

95 where the flexibilities of ligand and receptor side chains are 96 sampled simultaneously. Details of the docking method can be 97 found in ref. ${ }^{27}$ Briefly, a ligand rotamer library is generated in a 98 stochastic manner "on the fly". As a result, the sampling of ligand 99 conformations is treated in a unified way, as in the sampling of 100 protein side chains, which are modeled by a discrete set of con101 formations, i.e. rotamers. ${ }^{31,32}$ The docking protocol is composed 102 of two steps. First, representative ligand conformations are gener103 ated by clustering the stochastic rotamer library of each ligand. 104 Each representative ligand conformation is rapidly fitted into a 105 "smoothed" receptor pocket by turning off the van der Waals 106 repulsion between the ligand and the receptor side chains and 107 subsequent rigid-body docking. In the second step, fine-docking 108 is performed from each of the coarsely docked poses, where the 109 binding pose is minimized by iterative repacking of the rota110 mers of ligand and receptor side chains as well as ligand rigid111 body minimization. In the second fine-docking step, the van der 112 Waals repulsions between ligand and receptor side chains are 113 included. We use the MedusaScore ${ }^{33}$ scoring function to guide 114 the docking.
MedusaScore. MedusaScore ${ }^{33}$ is a physical force field- 115 based scoring function that describes the major physical inter- 116 actions between proteins and ligands, including the van der 117 Waals interaction $\left(E_{\mathrm{vdw}}\right)$, hydrogen bonding $\left(E_{\mathrm{hbond}}\right)$, solvation 118 $\left(E_{\text {solv }}\right)$, and electrostatics $\left(E_{\mathrm{es}}\right)$. The van der Waals interaction 119 parameters are adopted from the CHARMM19 force field. We 120 dampen the rapid increase of van der Waals repulsion between 121 overlapping atoms by linear interpolation of the repulsive 122 term of the Lennard-Jones potential. ${ }^{31}$ We use the distance 123 and orientation-dependent hydrogen bond model proposed 124 by Kortemme and Baker. ${ }^{34}$ We compute the solvation energy 125 using the EEF1 implicit solvent model proposed by Lazaridis and 126 Karplus. ${ }^{35}$ The electrostatic interaction is calculated between the 127 formal charges, including the charged residues of arginine, lysine, 128 glutamate, and aspartate in proteins, and identified charged 129 groups in the ligand. We used the distance-dependent dielectric 130 constant, $\sim r$, to model the screening effect. We also introduce a 131 solvent-accessibility-dependent weighting coefficient to model 132 the environmental dependence of the electrostatic interactions. ${ }^{36}{ }_{133}$ 
134 The total MedusaScore describing the binding is the linear sum 135 of all of these energy terms.

136

$$
E=W_{\mathrm{vdw}} E_{\mathrm{vdw}}+W_{\text {hbond }} E_{\mathrm{hbond}}+W_{\mathrm{solv}} E_{\mathrm{solv}}+W_{\mathrm{es}} E_{\mathrm{es}}
$$

137 Here, the weights $(W)$ were originally trained and deter138 mined for describing interamino acid interactions in high-resolution 139 protein structures. ${ }^{31}$ Notably, no protein-ligand data was used in 140 the development of MedusaScore, ${ }^{33}$ but the scoring function still 141 exhibits remarkable accuracy in both docking pose discrimination 142 and binding affinity prediction. Therefore, MedusaScore features 143 high transferability in both docking and virtual screening. ${ }^{27}$

144 Backbone Structural Ensemble Selection. We use the 145 "Sequence Similarity" search functionality included in the pro146 tein databank ${ }^{37}$ Web site (www.rcsb.org) to identify all solved 147 crystal structures of a protein. Given the reference PDB struc148 tures by the CSAR2011 organizers for chk1 (PDB ID: 2e9n), 149 erk2 (PDB ID: 3i5z), lpxc (PDB ID: 3p3e), and urokinase 150 (PDB ID: lowe) kinases, we identified 62, 10, 4, and 4 solved 151 structures, respectively. Because these ensembles were relatively 152 small and many backbone conformations were very close to each 153 other, we simply aligned all known structures of each kinase and 154 visually identified the representative backbone structures that 155 represented all possible backbone variations in the ligand-binding 156 pocket of those structures using PyMol (www.pymol.org). For 157 large backbone ensembles and also for the purpose of automation, 158 the representative backbones can be selected using clustering 159 analysis.

160 Clustering. We cluster the ligand poses by root-mean-square 161 deviation (RMSD). Here, we compute the RMSD between two 162 ligands after aligning the two receptors. During the RMSD 163 calculation, we also consider the symmetry of atoms-where a 164 symmetric rotation does not change the physiochemical property 165 of the ligand, such as benzene ring flipping - by taking the lowest 166 deviation among all such symmetric transformations. We use a 167 hierarchical clustering program, oc (www.compbio.dundee.ac.uk/ 168 downloads/oc), to group similar poses using a cutoff distance 169 of $2.5 \AA$ A A hierarchical clustering algorithm iteratively joins the 170 two closest clusters into one cluster according to the distances 171 between two clusters. The "cluster distance" is computed based 172 on all pairwise distances between elements of the two corre173 sponding clusters, which can be the minimum, maximum, or the 174 mean of all these values. In this study, we use the mean to com175 pute the distance between two clusters.

176 Ranking of Clusters. We use two different ranking 177 approaches to rank the clusters. In the first approach, we simply 178 calculate the average MeduaScore

179

$$
\langle E\rangle_{\mathrm{c}}=1 / n_{\mathrm{c}} \sum_{i} E_{i}
$$

180 Here, $n_{\mathrm{c}}$ is the cluster size and $E_{i}$ is the MedusaScore of pose $181 i$ within a cluster $\mathrm{c}$. In the second approach, we compute the 182 effective free energy of each cluster

183

$$
F_{\mathrm{c}}=\sum_{i} E_{i} \exp \left(-\beta E_{i}\right) / \sum_{i} \exp \left(-\beta E_{i}\right)-k_{\mathrm{B}} T \ln \left(n_{\mathrm{c}}\right)
$$

184 Here, $\beta$ is the reciprocal of $k_{\mathrm{B}} T, \sim 0.6 \mathrm{kcal} / \mathrm{mol}$, which corre185 sponds to the thermal fluctuation energy at room temperature 186 (300 K).

\section{RESULTS}

188 There were four receptor targets in the CSAR2011 docking 189 benchmark exercise, including checkpoint kinase-1 (chk1), extracellular-signal-regulated kinase 2 (erk2), N-acetylglucosamine 190 deacetylase from Pseudomonas aeruginosa (lpxc), and urokinase. 191 The sequences and reference structures were given by reference to 192 existing experimental structures: chk1 (PDB ID: 2e9n), erk2 193 (PDB ID: 3i5z), lpxc (PDB ID: 3p3e), and urokinase (PDB ID: 194 lowe). For each target, ligands were provided in the smile format 195 (47 for chk1, 39 for erk2, 16 for lpxc, and 20 for urokinase; 196 www.csardock.org). The participants were allowed to use any 197 information and methods to model the conformations of the bound 198 complexes. For a subset of these ligands (Table 1), the crystal 199 structures of the ligand-receptor complexes were solved and were 200 used to compare with the blindly predicted poses submitted by 201 the participants.

Flexible Backbone Docking Using MedusaDock. We 203 use MedusaDock ${ }^{27}$ to generate ligand-binding poses in a given 204 receptor. MedusaDock models the flexibility of receptor side 205 chains in the pocket but not the receptor backbones (Methods 206 section). To incorporate backbone flexibility for the receptor 207 structures, especially kinases known for large backbone conforma- 208 tional changes upon ligand binding, ${ }^{6,8,29,30}$ we develop a simple flexi- 209 ble backbone approach (Figure 1). First, we construct an ensemble 210 of receptor backbone conformations. Because MedusaDock 211 already considers the full receptor side chain flexibility in the 212 binding pocket, which is found to tolerate small backbone varia- 213 tions, ${ }^{27}$ we only include a small number of backbone conforma- 214 tions that capture the backbone changes upon ligand binding. We 215 use known receptor structures solved under different conditions 216 to reconstruct the backbone ensemble for each target (Methods 217 section). The backbone ensemble of chk1 includes PDB struc- 218 tures of $2 \mathrm{e} 9 \mathrm{u}, 2 \mathrm{ghg}, 2 \mathrm{ym} 4$, and 3nle; the ensemble of erk2 219 includes PDB structures of 1tvo, 1wzy, 2ojg, 3i60, and 3sa0; 220 and the lpxc ensemble includes PDB structures of 2ves, 3p3e, 221 and $3 \mathrm{uly}$. We find significant backbone variation between the 222 various structures of these flexible receptors (Figure 2). We use 223

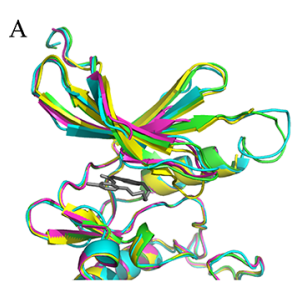

chk1

$2 \mathrm{e} 9 \mathrm{u}$

$2 \mathrm{ghg}$

$2 \mathrm{ym} 4$

3 nlb
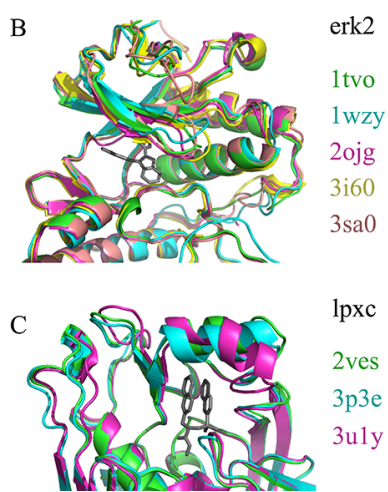

Figure 2. Multi-backbone ensembles for flexible backbone docking. The backbone structures in cartoon representation with different colors are selected from the protein databank for different CSAR2011 receptor targets: (A) chk1, (B) erk2, and (C) lpxc. The PDB IDs for the selected backbone structure are colored accordingly. 


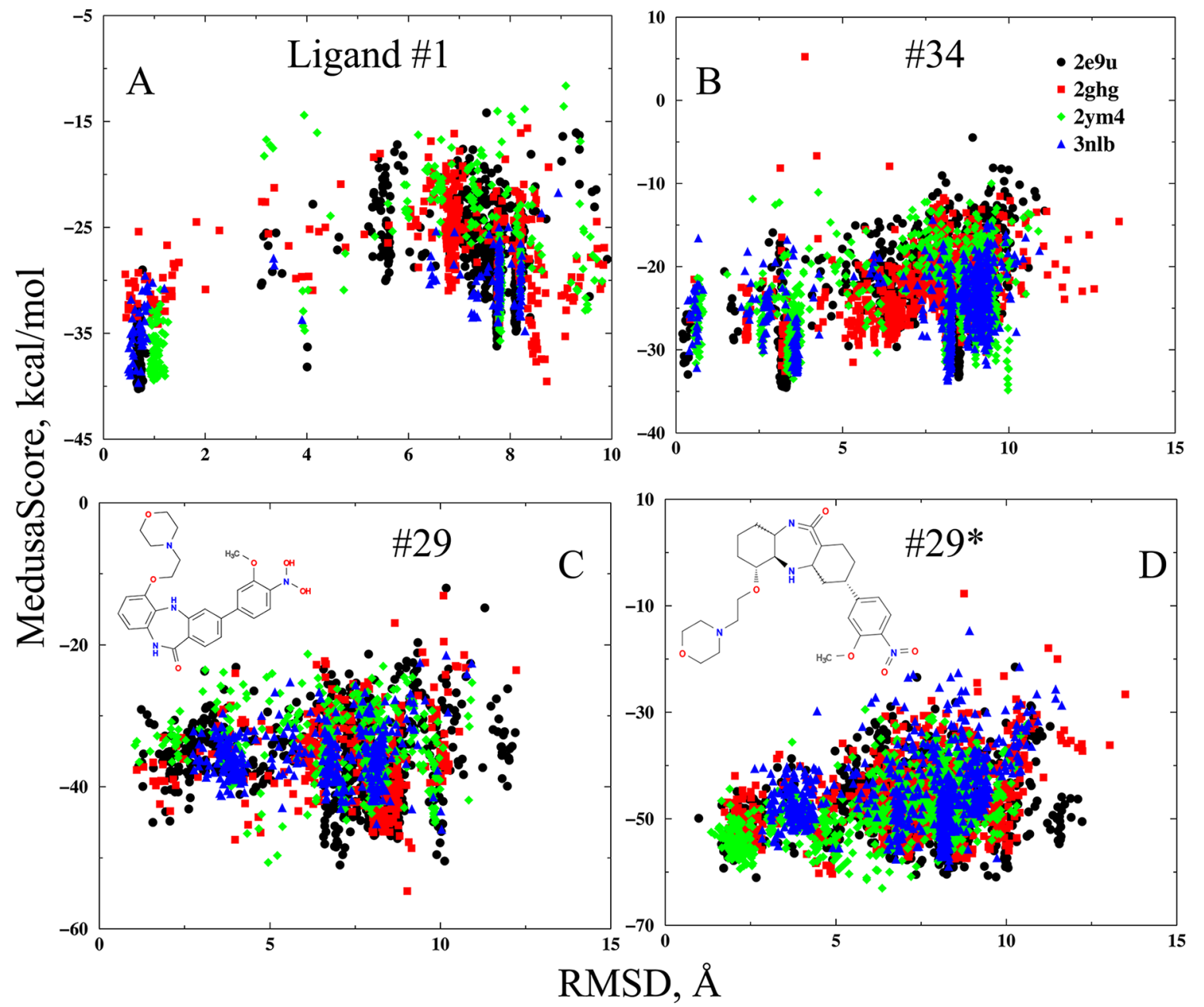

Figure 3. Scatter plot of MedusaScore versus RMSD for chk1 ligand poses. The symbols with different colors denote docking poses generated with different backbone conformations as shown in the legend. Panel (A) and (B) correspond to docking results of ligand \#1 and \#34, respectively. (C) The results for the docking of ligand \#29 that were initially given, which turned out to be different from the released structure. Panel (D) corresponds to the docking result of the actual ligand \#29, \#29*. The two chemical structures of ligand \#29 are given in the corresponding inserts.

224 only one backbone structure for urokinase (lowe) because all 225 known structures have very similar backbone structures.

226 Second, for each receptor backbone structure, we perform 227100 independent MedusaDock docking simulations. Depending 228 on the number of degrees of freedom of the input ligand, each 229 MedusaDock run generates several poses ${ }^{27}$ and takes on aver230 age approximately 3-5 min on an Intel $2.6 \mathrm{GHz}$ Xeon proces231 sor. All calculations can be done in parallel. Next, we rank all 232 poses for a given receptor backbone conformation according to 233 MedusaScore (Methodssection; eq 1). We collect the top $N_{\mathrm{p}}$ 234 poses for a given receptor backbone structure and assemble all 235 selected poses $\left(N_{\mathrm{b}} N_{\mathrm{p}}\right)$ from $N_{\mathrm{b}}$ backbone structures into a 236 single ensemble for further clustering (Figure 1). Clustering is 237 based on the RMSD between all pairs of poses, the calculation 238 time of which is proportional to $\left(N_{\mathrm{b}} N_{\mathrm{p}}\right)^{2}$. During the CSAR2011 239 exercise, we restricted $N_{\mathrm{p}} N_{\mathrm{b}}$ to approximately 500 total poses. We 240 group similar poses using a hierarchical clustering approach with a 241 cutoff RMSD of $2.5 \AA$ (Methodssection), and each cluster is 242 ranked according to the MedusaScore of poses within the cluster 243 (Methodssection). The centroid poses of the top three clusters 244 were submitted as CSAR2011 blind predictions.

245 Effective Selection of Native Poses Using Free Energy. 246 The CSAR2011 organizers allowed submission of more than one set of predicted poses, so that specific hypotheses can be 247 tested. We tested two different approaches to score and rank 248 the pose clusters (Methodssection). In the first approach, we 249 simply score the cluster by the average MedusaScore (eq 2). In 250 the second approach, we compute the effective free energy of 251 the cluster, where the average potential energy is computed as 252 the Boltzmann-weighted average of the MedusaScore, and the 253 entropy contribution is computed as the logarithm of the cluster 254 size (eq 3). We find that the scoring and ranking using the free 255 energy outperforms that by the average energy (Table 1). In the 256 case of free energy ranking, the lowest RMSD of the predicted 257 three poses is within $2.5 \AA$ for 28 out of 35 (80\%) targets. The 258 success rate of the predictions computed from the average energy 259 is 26 out of 35 . Therefore, scoring and ranking the clusters by the 260 proposed free energy is a more accurate way to select near-native 261 poses.

262

Docking with Multiple Backbone Conformations 263 Enriches Native-Like Poses. Our simple flexible backbone 264 docking approach is composed of independent MedusaDock 265 docking simulations with a set of predetermined backbone confor- 266 mations, and thus, the number of calculations is proportional to 267 the number of structures used. With ensemble docking, we sacri- 268 fice additional required computational time in exchange for 269 


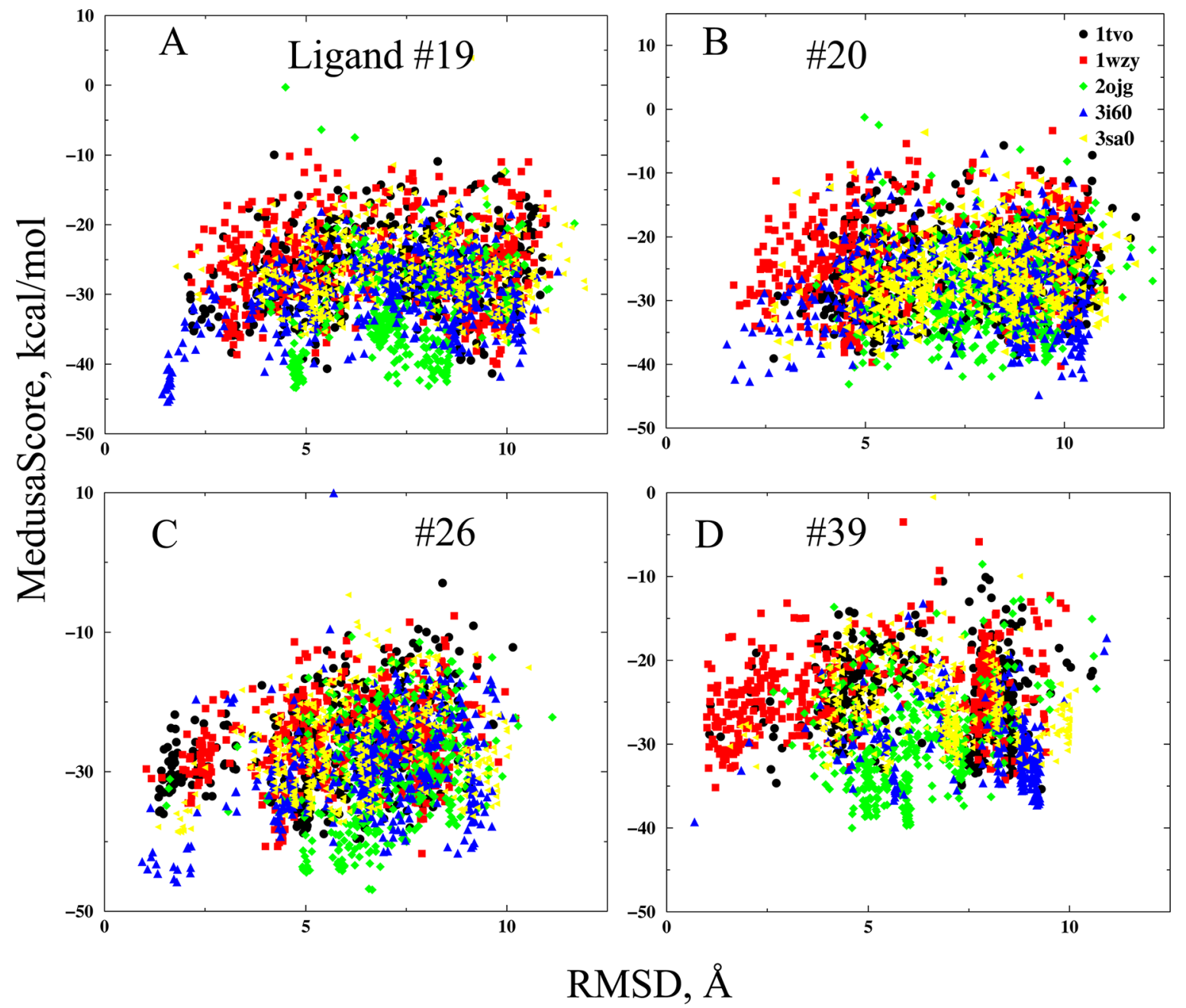

Figure 4. Scatter plot of MedusaScore versus RMSD for erk2 ligand poses. The symbols with different colors denote docking poses generated with different backbone conformations as shown in the legend. Four of the seven success cases are included: ligand \#19 (A), \#20 (B), \#26(C), and \#39 (D).

270 improved prediction accuracy. Next, we discuss the results of 271 docking for each receptor.

272 chk1. Bound structures have been solved experimentally for 273 chk1 with 14 different ligands (Table 1). Only in two cases 274 (ligand \#29 and \#34) do our free energy-ranked predictions not 275 succeed in identifying near-native poses. To illustrate the effect 276 of input backbone conformation on pose prediction, we present 277 in Figure 3 the scatter plot of MedusaScore versus RMSD for 278 the poses generated with different backbone conformations. 279 For example, in the case of ligand \#1 (Figure 3A), the poses 280 generated from three backbone conformations ( $2 \mathrm{e} 9 \mathrm{u}, 2 \mathrm{ym} 4$, 281 and 3nlb) feature a funnel-like binding/docking energy land282 scape, where the native-native poses have the lowest (most 283 favorable) MedusaScores. However, in the case of backbone 284 conformation of $2 \mathrm{ghg}$, the generated near-native poses have 285 higher (less favorable) MedusaScores than other decoy poses. 286 Taken together, MedusaDock simulations with multiple back287 bones enable the accurate prediction of a near-native pose as 288 the top-ranked pose for ligand \#1 (Table 1). For the failed case of 289 ligand \#34, near-native poses were sampled for all input backbone 290 conformations but all had higher MedusaScores than the decoy 291 poses (Figure 3B). Interestingly, near-native poses of ligand \#34 292 were top-ranked when utilizing the scoring method with average 293 energy (Table 1). Additionally, we noticed that the chemical structure of ligand \#29 in the final released structure is different 294 from that provided by the input smile (inserts of Figure 3C,D). We 295 therefore performed flexible docking simulations for the revised 296 structure of ligand \#29 after its final release. Although dock- 297 ing to the backbone of 3nlb did not sample near-native states 298 (Figure 3D), the revised ligand structure enabled sufficient sampl- 299 ing of near-native poses when docked to other backbone struc- 300 tures (Figure 3D), achieving a near-native pose as the second- 301 ranked pose (Table 1).

erk2. Twelve erk2-ligand complex structures have been solved 303 experimentally (Table 1). Our method recapitulated near-native 304 poses for seven out of twelve cases. The relatively low success 305 rate compared to chk1 may be a result of high backbone flexi- 306 bility, which is manifested even in the success cases (Table 1; 307 Figure 4). For example, near-native poses were sampled by 308 docking only a small number of backbone conformations (e.g., 309 Figure 4A,B) as compared to chk1 (Figure 3). In other cases, the 310 sampled near-native poses did not have clear separation from 311 decoy poses in terms of MedusaScore (Figure 4B,D). However, 312 using the clustering and ranking approach, we are able to select 313 near-native poses from many decoys in the latter cases. These 314 results (Figure 4), as well as those for chk1 (Figure 3), highlight 315 the importance of incorporating multiple backbone conformations 316 in the sampling of near-native poses with low MedusaScores. $\quad 317$ 


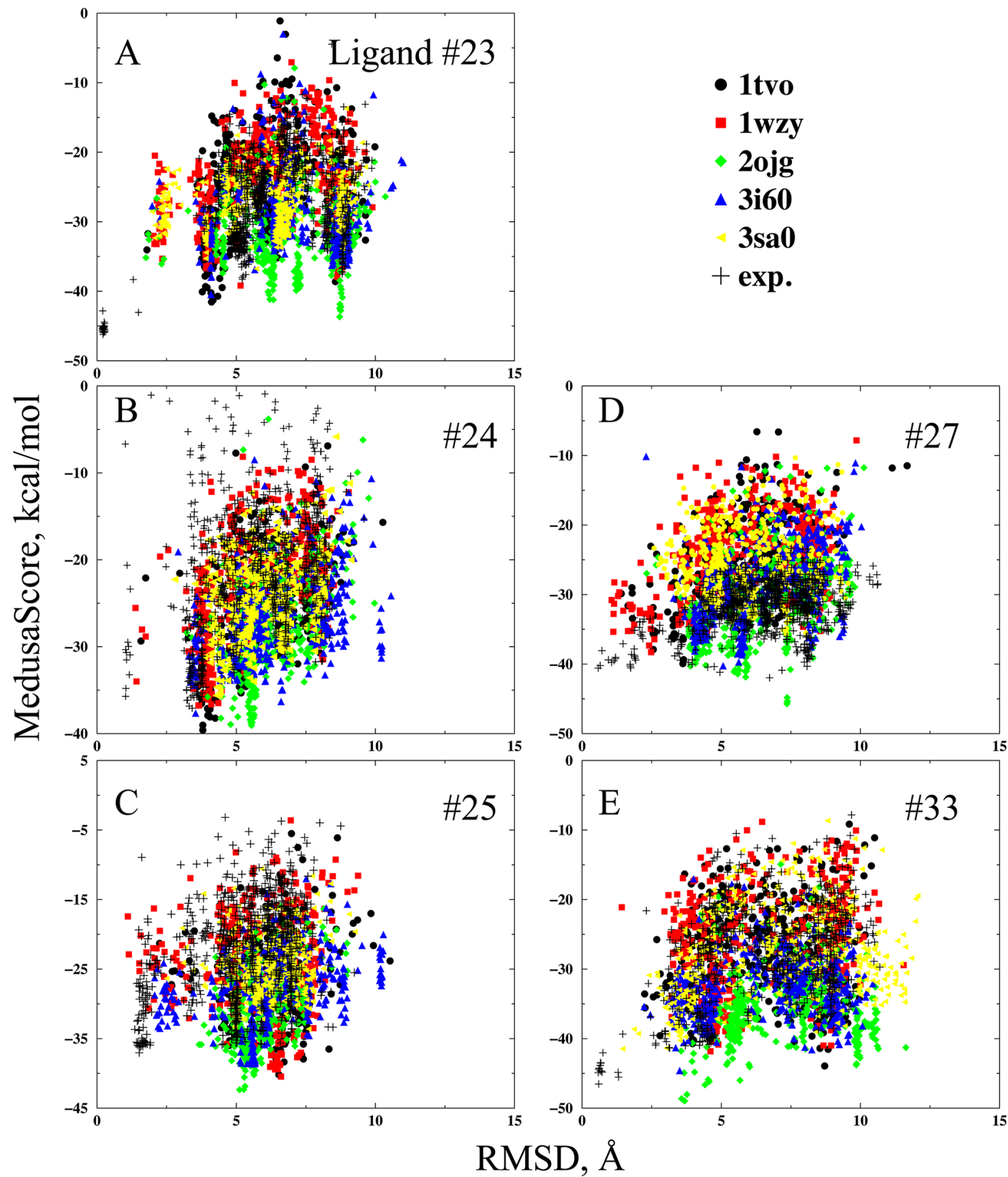

Figure 5. Scatter plot of MedusaScore versus RMSD for erk2 ligand poses. The symbols with different colors denote docking poses generated with different backbone conformations as shown in the legend. Five of the challenging cases are included: ligand \#23 (A), \#24 (B), \#25(C), \#27(D), and \#33 (E).

318 In five challenging cases (Figure 5, filled symbols correspond 319 to blind docking results for CSAR2011), the near-native states 320 were either rarely sampled (Figure 5B,E) or sampled but with 321 significantly less favorable MedusaScores than decoys (Figure $3225 \mathrm{~A}, \mathrm{C}, \mathrm{D})$. We postulate that the failure to capture near-native 323 states with low MedusaScores is a result of insufficient sampl324 ing of backbone conformations in the receptor ensemble. The 325 question is whether docking with the released cocrystallized 326 backbone structure can enrich the near-native poses with low 327 MedusaScores? Therefore, we performed posterior MedusaDock 328 simulations with the released backbone conformation. Indeed, the 329 docking with experimentally determined cocrystallized backbone conformations enables the sampling of near-native poses in all 330 challenging cases (Figure 5, plus symbols). If the cocrystallized 331 backbone structures were included in the predetermined back- 332 bone ensemble, the near-native poses of these challenging cases 333 could have been selected by our clustering and ranking method 334 as demonstrated by previous examples (Figures 3,4). Hence, 335 the results of these difficult cases suggest that the sampling 336 of backbone conformation in order to capture the backbone 337 changes upon binding of specific ligands remains a significant 338 challenge.

Ipxc and urokinase. The lpxc receptor has a zinc ion as the 340 coligand in all existing structures. We include the zinc ion as a 341 
342 fixed moiety during MedusaDock simulation. We recapitulate 343 near-native poses for both receptors (Table 1). In the case of 344 lpxc, all top-ranked poses correspond to near-native poses.

\section{DISCUSSION AND CONCLUSION}

346 In order to model receptor backbone changes upon ligand 347 binding, we develop a simple multibackbone docking approach 348 using MedusaDock. Because MedusaDock is able to model the 349 full receptor side chain flexibility, we construct a relatively small 350 ensemble of protein backbone conformations for the region 351 near the ligand-binding pocket. The flexible side chain docking 352 approach used by MedusaDock can tolerate small backbone 353 changes, as shown in the previous cross-docking benchmark. ${ }^{27}$ 354 For this reason, including a small number of backbone confor355 mations in the ensemble is most computationally efficient 356 because simulation time is directly proportional to the input 357 number of backbone conformations.

358 The major challenge in ensemble docking is to capture rele359 vant backbone changes upon ligand binding within the pre360 determined set of backbone configurations. For example, our pre361 constructed backbone ensembles for chk1, lpxc, and urokinase 362 (Table 1) are able to capture the corresponding backbone changes 363 upon binding of the given ligands, as suggested by the high 364 success rate of prediction of near-native poses $(\sim 100 \%)$. How365 ever, the prediction rate for erk2 kinase is significantly lower 366 because the backbone structures are not well sampled by the 367 constructed backbone ensemble (Figure 5). In the current study, 368 we have utilized crystallographically determined receptor struc369 tures solved in complex with different ligands and under different 370 conditions. Protein structural ensembles derived from solution 371 NMR also provides useful information about protein backbone 372 dynamics, ${ }^{38}$ which can be used for the flexible docking approach. 373 With the growing number of protein and protein-ligand com374 plex structures deposited into the Protein Data Bank, this approach 375 will have a broad application to drug screening. In cases where a 376 limited number of experimentally solved structures are available, 377 computational modeling of receptor backbone structures can be 378 performed via homology modeling, molecular dynamics, or normal 379 model analysis. The sampling of backbone changes as well as the 380 choice of the optimal number of backbone conformations to use in 381 MedusaDock ensemble docking simulations is a subject for further 382 studies.

383 We group similar poses using a clustering algorithm and 384 develop a free energy-like scoring method to rank clusters of poses. 385 The new score thus considers both the average MedusaScore of 386 each cluster as well as the cluster size in ranking poses (eq 3). We 387 use a Boltzmann-weighted average of MedusaScores within a cluster 388 to compute the final score, where a pose with a lower (more 389 favorable) MedusaScore has a higher weight. The second term with 390 logarithm of the cluster size also favor the large cluster with many 391 similar poses, corresponding to thermodynamic states with large 392 number of microstates and thus high entropy. Our clustering and 393 ranking approach allows us to select near-native poses even when 394 their scores are not obviously separated from those of decoy poses 395 (e.g., Figures 3D and 4B,D). As the result, we are able to predict the 396 near-native poses for 28 out of 35 ligands, which corresponds to the 397 highest success rate of near-native pose predictions (<2.5 ̊̊ RMSD) 398 in the CSAR2011 docking benchmark exercise. We expect a broach 399 application of our fully flexible docking approach in pose prediction 400 for both biological study as well as rational drug design.

\section{AUTHOR INFORMATION}

401

\section{Corresponding Author}

*E-mail: dokh@med.unc.edu.

Notes

The authors declare no competing financial interest.

\section{ACKNOWLEDGMENTS}

406

We thank Elizabeth Proctor for critical reading of the manuscript. 407 The calculations were performed on the Killdevil high perform- 408 ance cluster maintained by ITS at UNC. This work was supported 409 by GM080742 (to N.V.D.).

\section{REFERENCES}

411

(1) Leach, A. R.; Shoichet, B. K.; Peishoff, C. E. Prediction of 412 protein-ligand interactions. Docking and scoring: Successes and gaps. 413 J. Med. Chem. 2006, 49, 5851-5855.

414

(2) Sousa, S. F.; Fernandes, P. A.; Ramos, M. J. Protein-ligand 415 docking: Current status and future challenges. Proteins: Struct., Funct., 416 Bioinf. 2006, 65, 15-26.

417

(3) Carlson, H. A.; McCammon, J. A. Accommodating protein 418 flexibility in computational drug design. Mol. Pharmacol. 2000, 57, 419 213-218.

(4) Teague, S. J. Implications of protein flexibility for drug discovery. 421 Nat. Rev. Drug Discovery 2003, 2, 527-541.

(5) Teodoro, M. L.; Kavraki, L. E. Conformational flexibility models 423 for the receptor in structure based drug design. Curr. Pharm. Des. 424 2003, 9, 1635-1648.

425

(6) Schindler, T.; Bornmann, W.; Pellicena, P.; Miller, W. T.; 426 Clarkson, B.; Kuriyan, J. Structural mechanism for STI-571 inhibition 427 of abelson tyrosine kinase. Science 2000, 289, 1938-1942.

428

(7) Koska, J.; Spassov, V. Z.; Maynard, A. J.; Yan, L.; Austin, N.; 429 Flook, P. K.; Venkatachalam, C. M. Fully automated molecular 430 mechanics based induced fit protein-ligand docking method. J. Chem. 431 Inf. Model. 2008, 48, 1965-1973.

432

(8) May, A.; Zacharias, M. Protein-ligand docking accounting for 433 receptor side chain and global flexibility in normal modes: Evaluation 434 on kinase inhibitor cross docking. J. Med. Chem. 2008, 51, 3499-3506. 435

(9) Meiler, J.; Baker, D. ROSETTALIGAND: Protein-small 436 molecule docking with full side-chain flexibility. Proteins: Struct., 437 Funct., Bioinf. 2006, 65, 538-548.

(10) Nabuurs, S. B.; Wagener, M.; de Vlieg, J. A flexible approach to 439 induced fit docking. J. Med. Chem. 2007, 50, 6507-6518. 440

(11) Rueda, M.; Bottegoni, G.; Abagyan, R. Consistent improvement 441 of cross-docking results using binding site ensembles generated with 442 elastic network normal modes. J. Chem. Inf. Model. 2009, 49, 716-725. 443

(12) Barril, X.; Morley, S. D. Unveiling the full potential of flexible 444 receptor docking using multiple crystallographic structures. J. Med. 445 Chem. 2005, 48, 4432-4443.

(13) Sheridan, R. P.; McGaughey, G. B.; Cornell, W. D. Multiple 447 protein structures and multiple ligands: Effects on the apparent 448 goodness of virtual screening results. J. Comput.-Aided Mol. Des. 2008, 449 22, 257-265.

(14) Knegtel, R. M.; Kuntz, I. D.; Oshiro, C. M. Molecular docking 451 to ensembles of protein structures. J. Mol. Biol. 1997, 266, 424-440. 452

(15) Damm, K. L.; Carlson, H. A. Exploring experimental sources of 453 multiple protein conformations in structure-based drug design. J. Am. 454 Chem. Soc. 2007, 129, 8225-8235.

(16) Cheng, L. S.; Amaro, R. E.; Xu, D.; Li, W. W.; Arzberger, P. W.; 456 McCammon, J. A. Ensemble-based virtual screening reveals potential 457 novel antiviral compounds for avian influenza neuraminidase. J. Med. 458 Chem. 2008, 51, 3878-3894.

(17) Soliva, R.; Gelpi, J. L.; Almansa, C.; Virgili, M.; Orozco, M. 460 Dissection of the recognition properties of p38 MAP kinase. 461 Determination of the binding mode of a new pyridinyl-heterocycle 462 inhibitor family. J. Med. Chem. 2007, 50, 283-293.

(18) Karplus, M. Molecular dynamics of biological macromolecules: 464 A brief history and perspective. Biopolymers 2003, 68, 350-358. 465 
466 (19) Karplus, M.; Kuriyan, J. Molecular dynamics and protein 467 function. Proc. Natl. Acad. Sci. U.S.A. 2005, 102, 6679-6685.

468 (20) Fan, H.; Irwin, J. J.; Webb, B. M.; Klebe, G.; Shoichet, B. K.; 469 Sali, A. Molecular docking screens using comparative models of 470 proteins. J. Chem. Inf. Model. 2009, 49, 2512-2527.

471 (21) Keseru, G. M.; Kolossvary, I. Fully flexible low-mode docking: 472 Application to induced fit in HIV integrase. J. Am. Chem. Soc. 2001, 473 123, 12708-12709.

474 (22) Cavasotto, C. N.; Kovacs, J. A.; Abagyan, R. A. Representing 475 receptor flexibility in ligand docking through relevant normal modes. J. $476 \mathrm{Am}$. Chem. Soc. 2005, 127, 9632-9640.

477 (23) Huang, S. Y.; Zou, X. Ensemble docking of multiple protein 478 structures: Considering protein structural variations in molecular 479 docking. Proteins: Struct., Funct., Bioinf. 2007, 66, 399-421.

480 (24) Kairys, V.; Gilson, M. K. Enhanced docking with the mining 481 minima optimizer: Acceleration and side-chain flexibility. J. Comput. 482 Chem. 2002, 23, 1656-1670.

483 (25) Anderson, A. C.; O’Neil, R. H.; Surti, T. S.; Stroud, R. M. 484 Approaches to solving the rigid receptor problem by identifying a 485 minimal set of flexible residues during ligand docking. Chem. Biol. 486 2001, 8, 445-457.

487 (26) Davis, I. W.; Baker, D. RosettaLigand docking with full ligand 488 and receptor flexibility. J. Mol. Biol. 2009, 385, 381-392.

489 (27) Ding, F.; Yin, S.; Dokholyan, N. V. Rapid flexible docking using 490 a stochastic rotamer library of ligands. J. Chem. Inf. Model. 2010, 50, 491 1623-1632.

492 (28) Dunbrack, R. L., Jr.; Karplus, M. Backbone-dependent rotamer 493 library for proteins. Application to side-chain prediction. J. Mol. Biol. 494 1993, 230, 543-574.

495 (29) Bahar, I.; Erman, B.; Jernigan, R. L.; Atilgan, A. R.; Covell, D. G. 496 Collective motions in HIV-1 reverse transcriptase: Examination of 497 flexibility and enzyme function. J. Mol. Biol. 1999, 285, 1023-1037.

498 (30) Wang, Y. X.; Freedberg, D. I.; Yamazaki, T.; Wingfield, P. T.; 499 Stahl, S. J.; Kaufman, J. D.; Kiso, Y.; Torchia, D. A. Solution NMR 500 evidence that the HIV-1 protease catalytic aspartyl groups have 501 different ionization states in the complex formed with the asymmetric 502 drug KNI-272. Biochemistry 1996, 35, 9945-9950.

503 (31) Ding, F.; Dokholyan, N. V. Emergence of protein fold families 504 through rational design. PLoS Comput. Biol. 2006, 2, e85.

505 (32) Yin, S.; Ding, F.; Dokholyan, N. V. Eris: An automated 506 estimator of protein stability. Nat. Methods 2007, 4, 466-467.

507 (33) Yin, S.; Biedermannova, L.; Vondrasek, J.; Dokholyan, N. V. 508 MedusaScore: An accurate force field-based scoring function for virtual 509 drug screening. J. Chem. Inf. Model. 2008, 48, 1656-1662.

510 (34) Kortemme, T.; Morozov, A. V.; Baker, D. An orientation511 dependent hydrogen bonding potential improves prediction of 512 specificity and structure for proteins and protein-protein complexes. 513 J. Mol. Biol. 2003, 326, 1239-1259.

514 (35) Lazaridis, T.; Karplus, M. Effective energy function for proteins 515 in solution. Proteins: Struct., Funct., Bioinf. 1999, 35, 133-152.

516 (36) Matthew, J. B.; Gurd, F. R. Calculation of electrostatic 517 interactions in proteins. Methods Enzymol. 1986, 130, 413-436.

518 (37) Berman, H. M.; Westbrook, J.; Feng, Z.; Gilliland, G.; Bhat, T. 519 N.; Weissig, H.; Shindyalov, I. N.; Bourne, P. E. The Protein Data 520 Bank. Nucleic Acids Res. 2000, 28, 235-242.

521 (38) Best, R. B.; Lindorff-Larsen, K.; DePristo, M. A.; Vendruscolo, $522 \mathrm{M}$. Relation between native ensembles and experimental structures of 523 proteins. Proc. Natl. Acad. Sci. U.S.A. 2006, 103, 10901-10906. 